\title{
SEGMENTATION OF FLUORESCENCE MICROSCOPY IMAGES USING THREE DIMENSIONAL ACTIVE CONTOURS WITH INHOMOGENEITY CORRECTION
}

\author{
Soonam Lee $\quad$ Paul Salama $\quad$ Kenneth W. Dunn ${ }^{\ddagger} \quad$ Edward J. Delp ${ }^{\star}$
}

\author{
${ }^{\star}$ Video and Image Processing Laboratory \\ School of Electrical and Computer Engineering \\ Purdue University \\ West Lafayette, Indiana
}

\author{
${ }^{\dagger}$ Department of Electrical and \\ Computer Engineering \\ Indiana University \\ Indianapolis, Indiana
}

\author{
${ }^{\ddagger}$ Division of Nephrology \\ School of Medicine \\ Indiana University \\ Indianapolis, Indiana
}

\begin{abstract}
Image segmentation is an important step in the quantitative analysis of fluorescence microscopy data. Since fluorescence microscopy volumes suffer from intensity inhomogeneity, low image contrast and limited depth resolution, poor edge details, and irregular structure shape, segmentation still remains a challenging problem. This paper describes a nuclei segmentation method for fluorescence microscopy based on the use of three dimensional (3D) active contours with inhomogeneity correction. The correction information utilizes 3D volume information while addressing intensity inhomogeneity across vertical and horizontal directions. Experimental results demonstrate that the proposed method achieves better performance than other reported methods.
\end{abstract}

Index Terms - image segmentation, fluorescence microscopy, multiphoton microscopy

\section{INTRODUCTION}

Fluorescence microscopy was traditionally used for observing biological entities. It has recently become a powerful tool for studying cells because it enables the acquisition of image volumes deeper into tissue $[1,2,3]$. Since 3D biological structures are complex, manual quantification of these image volumes is a laborious or intractable process and therefore many image analysis tools have been devoted for fluorescence microscopy.

A first step in quantifying biological structures is segmentation. Fluorescence microscopy volumes have unique characteristics that complicate segmentation. In particular, microscopy volumes are anisotropic, with aberrations and distortions that vary in different directions $[4,5]$ giving rise to inhomogeneous intensities along the horizontal directions as well as poorer depth resolution, where the vertical resolution is approximately 3 to 4 times less than the horizontal resolution $[6,7]$. Since biological structures often consist of non rigid shapes with varying orientations, fluorescent probes frequently fail to delineate correct boundaries.

There have been various methods developed to segment biomedical images that address some of the above issues. Many segmentation methods are based on active contours [8] which minimize an energy/cost function iteratively while deforming an initial contour to fit objects of interest. There are also several variants of active contours. One of these is edge-based active contours $[8,9]$ that utilizes image gradient maps to aid in object identification. Typically, the results of edge-based active contours tend to be sensitive to image noise and rely heavily on the placement of the initial contour. To address these problems [10] employed an external energy term obtained by convolving a user controllable vector field kernel with an

This work was partially supported by a George M. O'Brien Award from the National Institutes of Health under grant NIH/NIDDK P30 DK079312. image edge map. Alternatively, in [11] the Poisson inverse gradient was used to obtain the initial contours to be used in segmentation.

Active contours have also been integrated with region-based approaches in an attempt to seek an energy equilibrium between foreground and background regions [12, 13]. Region-based methods tend to generate better results than edge-based active contours because region-based methods are relatively independent of the initial contour generation and are more robust against noise. In $[4,5]$ a 3D version of region-based active contours [12] which results in improved segmentation by incorporating 3D information was presented. More specifically, in [4] a coupled active surface which is a $3 \mathrm{D}$ version of multiphase level sets [13] that utilize an indicator function to account for only one background region was proposed to avoid multiple level sets overlapping. Similarly, in [14] the coupled active surfaces based method was improved by incorporating a watershed method and the Radon transform. These regionbased methods, however, fail to produce satisfactory results in images with intensity inhomogeneities [15, 16]. To address this [15] proposed a localized region-based active contour method that uses a new energy functional, means separation, and histogram separation to distinguish between foreground regions and inhomogeneous background regions. Similarly, a modified energy function was used in [17] to address image inhomogeneities. Additionally, [18] presented multiple retrospective inhomogeneity correction methods as preprocessing steps to improve segmentation results under inhomogeneous intensities. More recently, in [16] we utilized adaptive thresholding and vertical direction refinement followed by boundary fitting to segment microscopy volumes while taking into account intensity inhomogeneities. Alternatively, [19] implemented a 3D segmentation method with an energy function based on exponential B-splines, while [20] proposed combining edge-based and region-based energy functions to segment fluorescence microscopy images.

An alternative approach based on multidimensional segmentation using random seeds in combination with multi-resolution, multiscale, and region growing was proposed in [21] while a combination of midpoint analysis, shape-based function optimization, and a Marked Point Process (MPP) simulation to quantify nuclei was presented in [22]. Additionally, [23] described a new approach to segmentation by coupling image restoration and segmentation using a generalized linear model and Bregman divergence.

In this paper, we describe a method that segments nuclei in 3D microscopy volumes based on a combination of 3D region-based active contours and 3D inhomogeneity correction. Nuclear segmentation is a prerequisite step for a variety of cell cytometry approaches used in biological research. Prior work that incorporated 3D snakes did not account for intensity inhomogeneities $[4,5]$. The method described here extends $3 \mathrm{D}$ region-based active contour methods by using 3D inhomogeneity correction. Our datasets consist of 3D vol- 
umes of a rat kidney labeled with Hoechst 33342 collected using two-photon microscopy. The goal here is to account for intensity inhomogeneity while incorporating 3D information.

\section{PROPOSED METHOD}

The main idea of region-based active contours is to evolve a curve (2D) or a surface (3D) $\phi$ towards an object boundary by minimizing an energy function. The energy function used in 3D region-based active contours $[4,5]$, which is an extension of the $2 \mathrm{D}$ case [12], is given by:

$$
\begin{aligned}
E & =\lambda_{1} \int_{\text {in }\left(\phi_{z_{p}}\right)}\left|\mathcal{I}_{z_{p}}^{O}(\mathbf{x})-c_{1}\right|^{2} d \mathbf{x}+\lambda_{2} \int_{\text {out }\left(\phi_{z_{p}}\right)}\left|\mathcal{I}_{z_{p}}^{O}(\mathbf{x})-c_{2}\right|^{2} d \mathbf{x} \\
& +\mu \cdot \operatorname{Surface}\left(\phi_{z_{p}}(\mathbf{x})\right)
\end{aligned}
$$

where $\mathbf{x} \in \mathbb{R}^{3}, \mathcal{I}_{z_{p}}^{O}(\mathbf{x})$ is the $p^{\text {th }}$ image in a volume to be analyzed where $p \in\{1,2, \ldots, P\}, \phi_{z_{p}}(\mathbf{x})$ is a zero-level surface (Lipschitz function) of same size as $\mathcal{I}_{z_{p}}^{O}(\mathbf{x}), c_{1}$ is the average intensity inside $\phi_{z_{p}}(\mathbf{x}), c_{2}$ is the average intensity outside $\phi_{z_{p}}(\mathbf{x})$, and $\lambda_{1}, \lambda_{2}, \mu$ are weighting coefficients for each term, respectively.

The method adopted here extends a previous 3D region-based active contour method $[4,5]$ by taking into account $3 \mathrm{D}$ intensity inhomogeneities. This is achieved by utilizing a multiplicative model where the original volume $\mathcal{I}_{z_{p}}^{O}(\mathbf{x})$ is modeled as

$$
\mathcal{I}_{z_{p}}^{O}(\mathbf{x})=\mathcal{W}_{z_{p}}(\mathbf{x}) \circ \mathcal{I}_{z_{p}}^{C}(\mathbf{x})+\mathcal{N}_{z_{p}}(\mathbf{x})
$$

where $\mathcal{I}_{z_{p}}^{C}(\mathbf{x})$ is a homogeneous volume, $\mathcal{W}_{z_{p}}(\mathbf{x})$ is a $3 \mathrm{D}$ weight matrix referred to as the inhomogeneity field that accounts for the degree of intensity inhomogeneity at each voxel location, and $\mathcal{N}_{z_{p}}(\mathbf{x})$ is zero-mean 3D Gaussian noise. The o operator represents voxelwise multiplication (Hadamard product). Assuming $\mathcal{W}_{z_{p}}(\mathbf{x})$ is slowly varying, Eq (2) can be approximated as [17]

$$
\mathcal{I}_{z_{p}}^{O}(\mathbf{x}) \approx \begin{cases}\mathcal{W}_{z_{p}}(\mathbf{y}) c_{1}+\mathcal{N}_{z_{p}}(\mathbf{x}) & \text { when } \phi_{z_{p}}(\mathbf{x})>0 \\ \mathcal{W}_{z_{p}}(\mathbf{y}) c_{2}+\mathcal{N}_{z_{p}}(\mathbf{x}) & \text { when } \phi_{z_{p}}(\mathbf{x})<0\end{cases}
$$

where $\mathbf{y}$ is any point in $B(\mathbf{x}, \boldsymbol{\rho})$, the $3 \mathrm{D}$ spherical neighborhood of $\mathbf{x}$ with radius $\boldsymbol{\rho}$, and $c_{1}, c_{2}$ are determined by whether $\mathbf{x}$ is inside or outside of the surface $\phi_{z_{p}}(\mathbf{x})$. Specifically, if the location of $\mathbf{x}$ is inside $\phi_{z_{p}}(\mathbf{x}), c_{1}$ is chosen as a centroid. However, if the location of $\mathbf{x}$ is outside of $\phi_{z_{p}}(\mathbf{x}), c_{2}$ is taken as the centroid. Using this relationship, a new energy function with a $3 \mathrm{D}$ kernel $\mathcal{K}$ is obtained:

$$
\begin{aligned}
E & =\int_{\Omega}\left(\lambda_{1} \int_{i n\left(\phi_{z_{p}}\right)} \mathcal{K}(\mathbf{y}-\mathbf{x}) \cdot\left|\mathcal{I}_{z_{p}}^{O}(\mathbf{x})-\mathcal{W}_{z_{p}}(\mathbf{y}) c_{1}\right|^{2} d \mathbf{x}\right. \\
& \left.+\lambda_{2} \int_{\text {out }\left(\phi_{z_{p}}\right)} \mathcal{K}(\mathbf{y}-\mathbf{x}) \cdot\left|\mathcal{I}_{z_{p}}^{O}(\mathbf{x})-\mathcal{W}_{z_{p}}(\mathbf{y}) c_{2}\right|^{2} d \mathbf{x}\right) d \mathbf{y} \\
& +\mu \cdot \operatorname{Surface}\left(\phi_{z_{p}}(\mathbf{x})\right) .
\end{aligned}
$$

Note that $\mathbf{y}$ is integrated over the entire image volume $\Omega$. Note also that the kernel function is chosen such that $\mathcal{K}(\mathbf{y}-\mathbf{x})=0$ when $\mathbf{y}$ is outside the neighborhood of $\mathbf{x}$ [24].

Since the kernel function $\mathcal{K}$ should account for a slow varying $\mathcal{W}_{z_{p}}$, we choose a modified normalized truncated 3D Gaussian function:

$$
\mathcal{K}(\mathbf{u})= \begin{cases}\frac{1}{C} e^{-\left|\frac{u_{x}^{2}}{2 \rho_{x}^{2}}+\frac{u_{y}^{2}}{2 \rho_{y}^{2}}+\frac{u_{z}^{2}}{2 \rho_{z}^{2}}\right|} & \text { when }|\mathbf{u}| \leq \boldsymbol{\rho} \\ 0 & \text { otherwise }\end{cases}
$$

where $\mathbf{u}=\left[u_{x}, u_{y}, u_{z}\right]^{T}$ and $\boldsymbol{\rho}=\left[\rho_{x}, \rho_{y}, \rho_{z}\right]^{T}$. Due to the difference in resolutions across the horizontal and vertical directions, and since the vertical resolution is approximately 4 times less than the horizontal resolution, we alter the neighborhood $B(\mathbf{x}, \boldsymbol{\rho})$ by setting $\rho_{x}=\rho_{y}=4 \rho_{z}$. In addition, $C$ is chosen to be a normalizing constant so that $\int \mathcal{K}(\mathbf{u}) d \mathbf{u}$ is always 1 .

Utilizing Heaviside's function, $H(\cdot)$, the Dirac delta function, $\delta(\cdot)$, and swapping the order of the integrals, Eq (4) can be rewritten as [12]:

$$
\begin{aligned}
& E=\lambda_{1} \int_{\Omega}\left(\left(\mathcal{I}^{O}\right)^{2} \circ 1_{\mathcal{K}}-2 \mathcal{I}^{O} \circ(\mathcal{W} * \mathcal{K}) c_{1}+\left(\mathcal{W}^{2} * \mathcal{K}\right) c_{1}^{2}\right) H(\phi) d \mathbf{x} \\
& +\lambda_{2} \int_{\Omega}\left(\left(\mathcal{I}^{O}\right)^{2} \circ 1_{\mathcal{K}}-2 \mathcal{I}^{O} \circ(\mathcal{W} * \mathcal{K}) c_{2}+\left(\mathcal{W}^{2} * \mathcal{K}\right) c_{2}^{2}\right)(1-H(\phi)) d \mathbf{x} \\
& +\mu \int_{\Omega} \delta(\phi)|\nabla \phi| d \mathbf{x}
\end{aligned}
$$

where $*$ is the $3 \mathrm{D}$ convolution operator and $1_{\mathcal{K}}(\mathbf{x})$ is a $3 \mathrm{D}$ volume of same size as $\mathcal{I}_{z_{p}}^{O}(\mathbf{x})$ whose entries are all 1 except near the volume boundary. More specifically, $1_{\mathcal{K}}(\mathbf{x})$ is obtained by convolving a $3 \mathrm{D}$ matrix of ones with $3 \mathrm{D}$ kernel $\mathcal{K}$. Note that both $c_{1}$ and $c_{2}$ are vectors with three elements $(3 \times 1$ vectors $)$. For brevity we have omitted the subscript $z_{p}$ and the explicit argument $\mathbf{x}$.

By minimizing the energy function shown in Eq (6) with respect to $\phi, \mathcal{W}, c_{1}$ and $c_{2}$, we can obtain the $3 \mathrm{D}$ segmentation result $(\phi)$ and an estimated 3D inhomogeneity field $(\mathcal{W})$.

One way to achieve this is to first minimize the energy function with respect to $c_{1}$ and $c_{2}$ for given $\phi$ and $\mathcal{W}$. Denoting the optimal values for $c_{1}$ and $c_{2}$ by $\hat{c_{1}}$ and $\hat{c_{2}}$, respectively, it can be shown that

$$
\hat{c_{1}}=\frac{\int_{i n(\phi)} \mathcal{I}^{O} \circ(\mathcal{W} * \mathcal{K}) d \mathbf{y}}{\int_{\text {in }(\phi)}\left(\mathcal{W}^{2} * \mathcal{K}\right) d \mathbf{y}}, \hat{c_{2}}=\frac{\int_{\text {out }(\phi)} \mathcal{I}^{O} \circ(\mathcal{W} * \mathcal{K}) d \mathbf{y}}{\int_{\text {out }(\phi)}\left(\mathcal{W}^{2} * \mathcal{K}\right) d \mathbf{y}}
$$

Having found $\hat{c_{1}}$ and $\hat{c_{2}}$ the next step is to minimize the energy function with respect to $\phi$ for given $c_{1}, c_{2}$, and $\mathcal{W}$. In this case the values used for $c_{1}$ and $c_{2}$ are $\hat{c_{1}}$ and $\hat{c_{2}}$, respectively. Since the partial differential equation (PDE) $\frac{\partial E}{\partial \phi}=0$ does not have a closed form solution, an approximate solution $\hat{\phi}$ can be obtained by iteratively using the Euler-Lagrange equation as follows [5]:

$$
\hat{\phi}_{\text {curr }}=\hat{\phi}_{\text {prev }}+\Delta t\left[\lambda_{2} f_{2}-\lambda_{1} f_{1}+\mu \operatorname{div}\left(\frac{\nabla \hat{\phi}_{\text {prev }}}{\left|\nabla \hat{\phi}_{\text {prev }}\right|}\right)\right]
$$

where $f_{1}$ and $f_{2}$ are expressed as

$$
\begin{aligned}
& f_{1}=\left(\mathcal{I}^{O}\right)^{2} \circ 1_{\mathcal{K}}-2 \mathcal{I}^{O} \circ(\mathcal{W} * \mathcal{K}) c_{1}+\left(\mathcal{W}^{2} * \mathcal{K}\right) c_{1}^{2} \\
& f_{2}=\left(\mathcal{I}^{O}\right)^{2} \circ 1_{\mathcal{K}}-2 \mathcal{I}^{O} \circ(\mathcal{W} * \mathcal{K}) c_{2}+\left(\mathcal{W}^{2} * \mathcal{K}\right) c_{2}^{2},
\end{aligned}
$$

respectively. $\hat{\phi}_{\text {prev }}$ and $\hat{\phi}_{\text {curr }}$ denote the previous and current estimates of $\phi$, respectively, $\Delta t$ a time step used to control the evolution speed of $\phi$, and $\mu$ the surface weight coefficient, chosen based on the size of the desired object to be detected. Higher $\mu$ values are used for detecting all objects whereas smaller $\mu$ values are used for detecting only larger objects [12]. Note that the term $\kappa=\operatorname{div}\left(\frac{\nabla \phi}{|\nabla \phi|}\right)$ is the curvature of the level set function $\phi$ and is expressed as [25]:

$$
\kappa=\frac{\left\{\begin{array}{r}
\phi_{x}^{2}\left(\phi_{y y}+\phi_{z z}\right)+\phi_{y}^{2}\left(\phi_{x x}+\phi_{z z}\right)+\phi_{z}^{2}\left(\phi_{x x}+\phi_{y y}\right) \\
-2\left(\phi_{x} \phi_{y} \phi_{x y}+\phi_{x} \phi_{z} \phi_{x z}+\phi_{y} \phi_{z} \phi_{y z}\right)
\end{array}\right\}}{\left(\phi_{x}^{2}+\phi_{y}^{2}+\phi_{z}^{2}\right)^{3 / 2}}
$$

Lastly, for given $c_{1}, c_{2}$, and $\phi, E$ is minimized with respect to the 3D inhomogeneity field $\mathcal{W}$. The optimal 3D inhomogeneity field, $\hat{\mathcal{W}}$, is the solution to the PDE arising from setting $\frac{\partial E}{\partial \mathcal{W}}=0$ and is given by

$$
\hat{\mathcal{W}}=\frac{\left(\mathcal{I}^{O} \circ \mathcal{J}^{(1)}\right) * \mathcal{K}}{\mathcal{J}^{(2)} * \mathcal{K}}
$$




\begin{tabular}{|c||c|c|c||c|c|c||c|c||c|c|c|c|}
\hline \multicolumn{1}{|c||}{} & \multicolumn{3}{c||}{ Dataset-II } & \multicolumn{3}{c||}{ Dataset-III } & \multicolumn{3}{c|}{ Dataset-IV } & \multicolumn{3}{c|}{ Dataset-V } \\
\hline Method & Accuracy & Type-I & Type-II & Accuracy & Type-I & Type-II & Accuracy & Type-I & Type-II & Accuracy & Type-I & Type-II \\
\hline 2Dac $[12]$ & $54.71 \%$ & $43.31 \%$ & $1.98 \%$ & $61.89 \%$ & $32.42 \%$ & $10.27 \%$ & $57.39 \%$ & $38.91 \%$ & $3.70 \%$ & $72.28 \%$ & $20.44 \%$ & $7.29 \%$ \\
\hline 2Dlac $[15]$ & $57.62 \%$ & $39.14 \%$ & $3.25 \%$ & $58.21 \%$ & $31.52 \%$ & $10.27 \%$ & $66.35 \%$ & $28.13 \%$ & $5.51 \%$ & $63.47 \%$ & $27.45 \%$ & $9.08 \%$ \\
\hline 2DacIC $[17]$ & $73.12 \%$ & $25.09 \%$ & $\mathbf{1 . 7 9 \%}$ & $80.35 \%$ & $15.19 \%$ & $\mathbf{4 . 4 6 \%}$ & $86.18 \%$ & $11.30 \%$ & $2.52 \%$ & $87.64 \%$ & $8.99 \%$ & $\mathbf{3 . 3 8 \%}$ \\
\hline 3Dac $[4,5]$ & $79.76 \%$ & $16.63 \%$ & $3.61 \%$ & $78.43 \%$ & $15.14 \%$ & $6.42 \%$ & $72.86 \%$ & $24.98 \%$ & $\mathbf{2 . 1 6 \%}$ & $81.58 \%$ & $12.57 \%$ & $5.86 \%$ \\
\hline 3Dsquassh $[23]$ & $88.72 \%$ & $8.57 \%$ & $2.71 \%$ & $85.32 \%$ & $5.96 \%$ & $8.73 \%$ & $83.35 \%$ & $14.28 \%$ & $2.37 \%$ & $83.22 \%$ & $13.01 \%$ & $3.77 \%$ \\
\hline $\begin{array}{c}\text { 3DacIC } \\
\text { proposed) }\end{array}$ & $\mathbf{9 1 . 8 7 \%}$ & $\mathbf{5 . 6 1 \%}$ & $2.53 \%$ & $\mathbf{8 9 . 6 5 \%}$ & $\mathbf{4 . 5 0 \%}$ & $5.85 \%$ & $\mathbf{8 7 . 7 1 \%}$ & $\mathbf{9 . 4 9 \%}$ & $2.80 \%$ & $\mathbf{8 9 . 1 0 \%}$ & $\mathbf{7 . 0 0 \%}$ & $3.90 \%$ \\
\hline
\end{tabular}

Table 1. Comparison of the performances of proposed and other segmentation methods using Dataset-II, III, IV, and V

where $\mathcal{J}^{(1)}=\int_{\text {in }(\phi)} c_{1} d \mathbf{y}+\int_{\text {out }(\phi)} c_{2} d \mathbf{y}$ and $\mathcal{J}^{(2)}=\int_{\text {in }(\phi)} c_{1}^{2} d \mathbf{y}+$ $\int_{\text {out }(\phi)} c_{2}^{2} d \mathbf{y}[17]$. Using the above solution it is possible to iteratively arrive at a segmentation result as described in "Method 1".

Note that $N_{I}$ denotes the number of (inner loop) iterations needed to arrive at a solution for $\phi$ as indicated by Eq (8), whereas $N_{O}$ is the number of (outer loop) iterations required to update $c_{1}, c_{2}$ and $\mathcal{W}$.

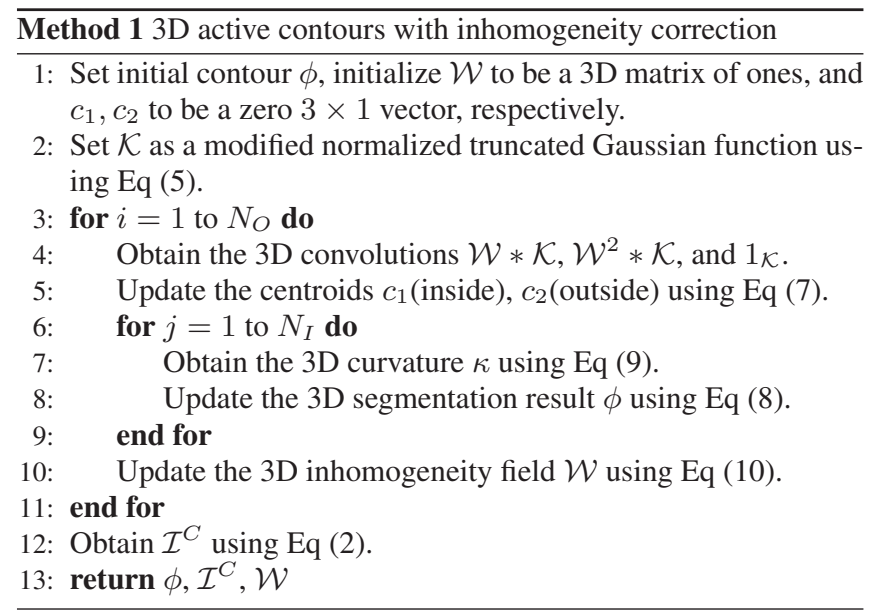

\section{EXPERIMENTAL RESULTS}

The performance of our method was tested on five different datasets: ${ }^{1}$ Dataset-I, II, III, IV, and $V$. The five datasets, which are volumes of rat kidney, consist of $512,36,41,38$, and 41 images, respectively each of size $512 \times 512$ pixels and are of 8 -bit/pixel resolution. Dataset- $I V$ and $V$ were collected from the same specimens as Dataset-II and III, respectively, but imaged in reverse directions.

The following values were used for the various parameters: $\Delta t=0.1, \lambda_{1}=\lambda_{2}=1, \rho_{x}=4, \mu=0.001 \cdot 255^{2}$. Since $\rho_{x}=\rho_{y}=4 \rho_{z}, \rho_{y}$ and $\rho_{z}$ are automatically determined once $\rho_{x}$ is set. Also, $N_{I}=20$ and $N_{O}=50$ so that the total number of iterations used in solving for $\phi$ was 1000 . For the initial contours, we chose multiple spheres of radius 10 distributed evenly to cover the entire volume. The segmentation results of the proposed method and corresponding inhomogeneity corrected images taken from various depth are shown in Figure 1.

As can be seen from the first row of Figure 1, the original images suffer from significant inhomogeneous intensities. More specifically, the intensities at the center are brighter than at the boundaries of the images. In spite of this, the proposed method is able to successfully capture nuclei close to the boundaries as shown in Figure 1.

\footnotetext{
${ }^{1}$ Dataset-I was provided by Malgorzata Kamocka of the Indiana Center for Biological Microscopy. Dataset-II, III, IV, and $V$ were provided by Tarek Ashkar of the Indiana University School of Medicine.
}

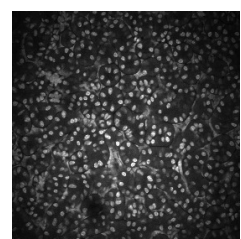

(a)

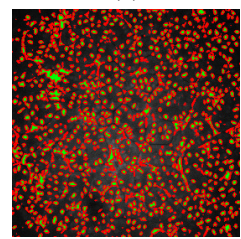

(d)

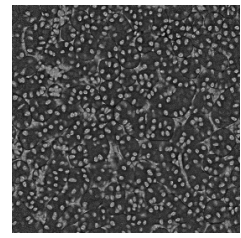

(g)

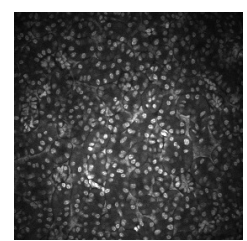

(b)

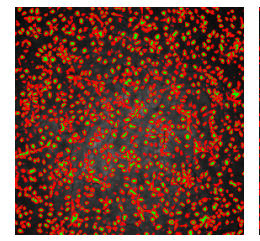

(e)

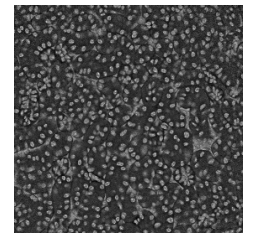

(h)

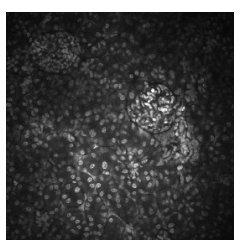

(c)

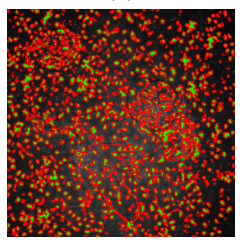

(f)

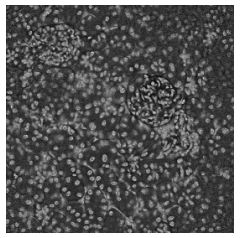

(i)
Fig. 1. Segmentation results and inhomogeneity corrected images at various depth for Dataset-I. Top row: Original $100^{t h}, 200^{t h}, 300^{t h}$ images. Middle row: Segmentation results overlaid onto original images (red: nuclei contours, green: nuclei regions). Bottom row: Corresponding inhomogeneity corrected images based on estimated 3D inhomogeneity field

For visualization purposes we highlight contours as red and their interiors as green. The last row of Figure 1 portrays the estimated inhomogeneity corrected images corresponding to the original images.

To evaluate the performance of the proposed method, images from Dataset-II, III, IV, and $V$ were manually segmented and used as groundtruth (Figures 2b, 3b). The accuracy, Type-I error, and Type-II error metrics were obtained for our method based on the groundtruth images. Here accuracy is defined to be the ratio of the number of correctly segmented nuclei pixels (true positive) and background pixels (true negative) to the total number of pixels. Type-I error (False alarm) is the ratio of the number of background pixels wrongly detected as nuclei (false positive) to the total number of pixels. Similarly, Type-II error (Missed) is the ratio of the number of nuclei pixels wrongly detected as background (false negative) to the total number of pixels.

The proposed method's performance is provided in Table 1 above where we have also included for comparison purposes the performance of five other techniques. In particular, we have included the 2D region-based active contours [12] (2Dac), 2D regionbased localized active contours [15] (2Dlac), 2D region-base active 


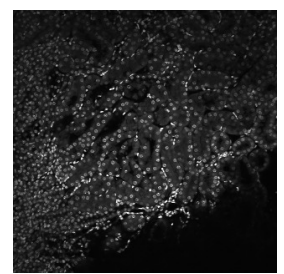

(a) Original

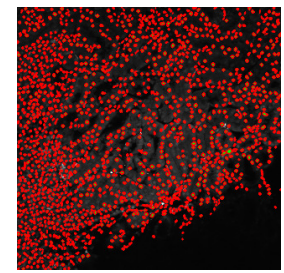

(b) Groundtruth

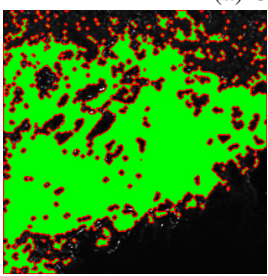

(c) $2 \mathrm{Dac}[12]$

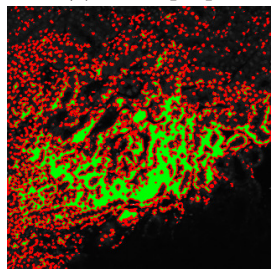

(f) $3 \mathrm{Dac}[4,5]$

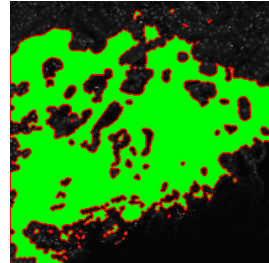

(d) 2 Dlac [15]

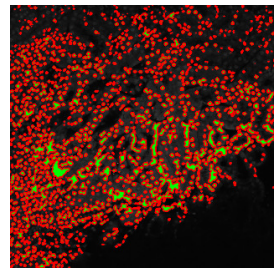

(g) 3Dsquassh [23]

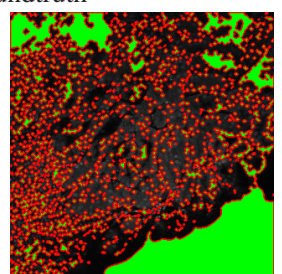

(e) $2 \mathrm{DacIC}$ [17]

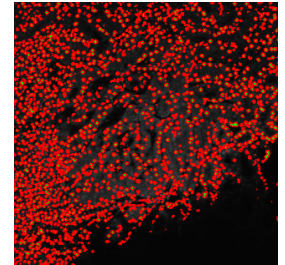

(h) 3DacIC (Proposed)
Fig. 2. Comparison of segmentation results (red: nuclei contours, green: nuclei regions) of the proposed method with other methods overlaid onto original image ( $7^{\text {th }}$ image of the Dataset-II)

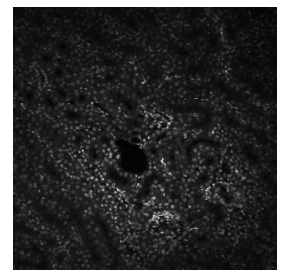

(a) Original

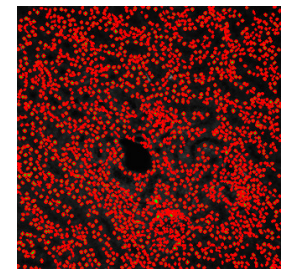

(b) Groundtruth

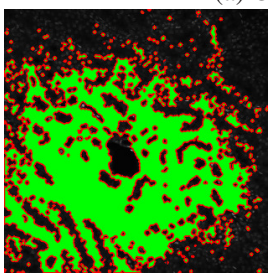

(c) $2 \mathrm{Dac}[12]$

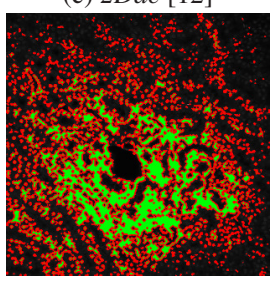

(f) $3 \mathrm{Dac}[4,5]$

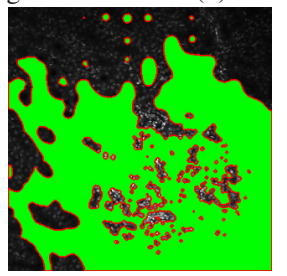

(d) 2 Dlac $[15]$

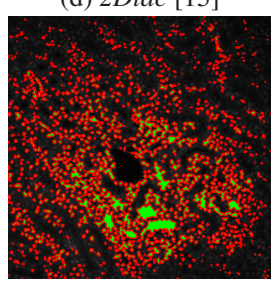

(g) 3Dsquassh [23]

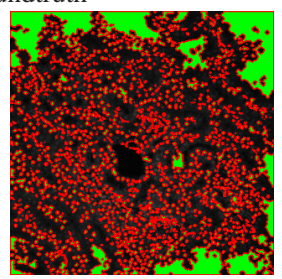

(e) $2 \mathrm{DacIC}$ [17]

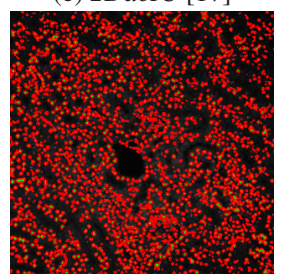

(h) 3DacIC (Proposed)
Fig. 3. Comparison of segmentation results (red: nuclei contours, green: nuclei regions) of the proposed method with other methods overlaid onto original image ( $16^{t h}$ image of the Dataset-III)

contours with inhomogeneity correction [17] (2DacIC), and 3D region-based active contours $[4,5](3 \mathrm{Dac})$. We denote our method by 3 DacIC ( $3 \mathrm{D}$ region-based active contours with inhomogeneity correction). For consistency, the same number and same sized cir- cles (2D)/spheres (3D) were used as initial contours in all the active contour methods. In addition, the same number of iterations were used. Finally, we also compared the performance of all these methods with a method described in [23] (3Dsquassh) using the default parameters setting in ImageJ.

As can be seen from Table 1, the proposed method outperformed the other methods in accuracy and Type-I error while exhibiting reasonably low Type-II error. 2DacIC and 3Dac had sometimes lower Type-II error than our method, but these methods suffered from high Type-I error, thus they often falsely detect nuclei. Similarly, 3Dsquass produced generally good accuracy and Type-II error but again suffered from high Type-I error.

For further comparison, we have included sample segmentation images of the results from Dataset-II and III produced by all the above mentioned techniques in Figure 2 and 3. The first row displays the original and groundtruth images, the second row exhibits segmentation results of $2 \mathrm{Dac}, 2 \mathrm{Dlac}, 2 \mathrm{DacIC}$, and the third row shows segmentation results of $3 D a c$, 3Dsquassh, and 3DacIC, respectively. As observed, the proposed method outperforms all other methods by properly identifying nuclei, especially nuclei close to the boundary. In comparison, $2 \mathrm{Dac}$ did not capture details at the center regions as well as boundary regions. $3 D a c$ had better performance than $2 D a c$ but failed to capture most nuclei located in the center. Although 3Dsquassh was capable of capturing more nuclei at the center than $3 D a c$, it still tended to group adjacent small nuclei as a single object as observed in Figure $2 \mathrm{~g}$ and $3 \mathrm{~g}$. In addition, both $3 \mathrm{Dac}$ and $3 D$ squassh missed many nuclei specifically at the boundary. In contrast, 2Dlac had poorer results than the others since each localized active contour utilized local information. To be more specific, each localized contour sometimes correctly identifies nuclei as foreground but sometimes not to the extent that the segmentation results were combined with background regions (Figure 2d, 3d). Although 2 DacIC did produce good segmentation results at the center regions, it also incorrectly segmented background regions at the boundary as nuclei (Figure 2e, 3e). This is a common problem for 2D based methods since they lack 3D information and their segmentation results are often inconsistent depthwise.

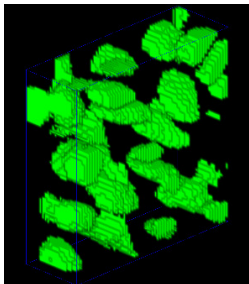

(a) Dataset-I

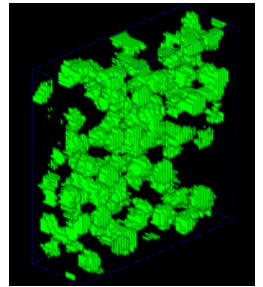

(b) Dataset-II

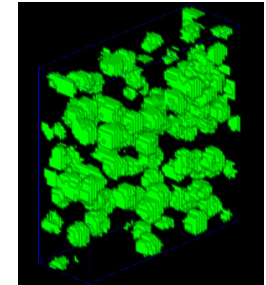

(c) Dataset-III
Fig. 4. 3D segmentation results for Dataset-I, II, and III (green: nuclei regions)

Lastly, Figure 4 portrays 3D segmentation results of various dataset using Voxx [26], a 3D visualization tool. For visualization purposes, each segmentation result was cropped into subvolumes $(60 \times 60 \times 20)$, respectively, which demonstrate that the proposed method successfully identified nuclei in 3D.

\section{CONCLUSION AND FUTURE WORK}

This paper has presented a 3D active contours with inhomogeneity correction method suitable for labeling nuclei in fluorescence microscopy volumes. Using 3D information, the proposed method can achieve better results than when using individual 2D slices. Future work will include quantifying the segmented nuclei and separating multiple overlapping nuclei. 


\section{REFERENCES}

[1] R. K. P. Benninger, M. Hao, and D. W. Piston, "Multi-photon excitation imaging of dynamic processes in living cells and tissues," Reviews of Physiology Biochemistry and Pharmacology, vol. 160, pp. 71-92, April 2008.

[2] D. W. Piston, "Imaging living cells and tissues by two-photon excitation microscopy," Trends in Cell Biology, vol. 9, no. 2, pp. 66-69, February 1999.

[3] P. T. C. So, C. Y. Dong, B. R. Masters, and K. M. Berland, "Two-photon excitation fluorescence microscopy," Annual Review of Biomedical Engineering, vol. 2, no. 1, pp. 399-429, August 2000.

[4] A. Dufour, V. Shinin, S. Tajbakhsh, N. Guillen-Aghion, J. C. Olivo-Marin, and C. Zimmer, "Segmenting and tracking fluorescent cells in dynamic 3-D microscopy with coupled active surfaces," IEEE Transactions on Image Processing, vol. 14, no. 9, pp. 1396-1410, September 2005.

[5] K. S. Lorenz, P. Salama, K. W. Dunn, and E. J. Delp, "Three dimensional segmentation of fluorescence microscopy images using active surfaces," Proceedings of the IEEE International Conference on Image Processing, pp. 1153-1157, September 2013, Melbourne, Australia.

[6] J. A. Rosado-Toro and J. J. Rodriguez, "Cell splitting using dynamic programming," Proceedings of the IEEE Southwest Symposium on Image Analysis and Interpretation, pp. 33-36, April 2012, Santa Fe, NM.

[7] C. L. Phillips, L. J. Arend, A. J. Filson, D. J. Kojetin, J. L. Clendenon, S. Fang, and K. W. Dunn, "Three-dimensional imaging of embryonic mouse kidney by two-photon microscopy," The American Journal of Pathology, vol. 158, no. 1, pp. 49-55, January 2001.

[8] M. Kass, A. Witkin, and D. Terzopoulos, "Snakes: Active contour models," International Journal of Computer Vision, vol. 1, no. 4, pp. 321-331, January 1988.

[9] V. Caselles, R. Kimmel, and G. Sapiro, "Geodesic active contours," International Journal of Computer Vision, vol. 22, no. 1, pp. 61-79, February 1997.

[10] B. Li and S. T. Acton, "Active contour external force using vector field convolution for image segmentation," IEEE Transactions on Image Processing, vol. 16, no. 8, pp. 2096-2106, August 2007.

[11] B. Li and S. T. Acton, "Automatic active model initialization via Poisson inverse gradient," IEEE Transactions on Image Processing, vol. 17, no. 8, pp. 1406-1420, August 2008.

[12] T. F. Chan and L. A. Vese, "Active contours without edges," IEEE Transactions on Image Processing, vol. 10, no. 2, pp. 266-277, February 2001.

[13] L. A. Vese and T. F. Chan, "A multiphase level set framework for image segmentation using the Mumford and Shah model," International Journal of Computer Vision, vol. 50, no. 3, pp. 271-293, December 2002.

[14] O. Dzyubachyk, W. A. van Cappellen, J. Essers, W. J. Niessen, and E. Meijering, "Advanced level-set-based cell tracking in time-lapse fluorescence microscopy," IEEE Transactions on Medical Imaging, vol. 29, no. 3, pp. 852-867, March 2010.
[15] S. Lankton and A. Tannenbaum, "Localizing region-based active contours," IEEE Transactions on Image Processing, vol. 17, no. 11, pp. 2029-2039, November 2008.

[16] S. Lee, P. Salama, K. W. Dunn, and E. J. Delp, "Boundary fitting based segmentation of fluorescence microscopy images," Proceedings of the IS\&T/SPIE Conference on Imaging and Multimedia Analytics in a Web and Mobile World 2015, pp. 940805-1-10, February 2015, San Francisco, CA.

[17] C. Li, R. Huang, Z. Ding, C. Gatenby, D. N. Metaxas, and J. C. Gore, "A level set method for image segmentation in the presence of intensity inhomogeneities with application to MRI,' IEEE Transactions on Image Processing, vol. 20, no. 7, pp. 2007-2016, July 2011.

[18] D. Tomazevic, B. Likar, and F. Pernus, "Comparative evaluation of retrospective shading correction methods," Journal of Microscopy, vol. 208, no. 3, pp. 212-223, December 2002.

[19] D. Schmitter, C. Gaudet-Blavignac, D. Piccini, and M. Unser, "New parametric 3D snake for medical segmentation of structures with cylindrical topology," Proceedings of the IEEE International Conference on Image Processing, pp. 276-280, September 2015, Quebec City, Canada.

[20] A. Badoual, D. Schmitter, and M. Unser, "Locally refinable parametric snakes," Proceedings of the IEEE International Conference on Image Processing, pp. 354-358, September 2015, Quebec City, Canada.

[21] G. Srinivasa, M. C. Fickus, Y. Guo, A. D. Linstedt, and J. Kovacevic, "Active mask segmentation of fluorescence microscope images," IEEE Transactions on Image Processing, vol. 18, no. 8, pp. 1817-1829, August 2009.

[22] N. Gadgil, P. Salama, K. W. Dunn, and E. J. Delp, "Nuclei segmentation of fluorescence microscopy images based on midpoint analysis and marked point process," Proceedings of the IEEE Southwest Symposium on Image Analysis and Interpretation, pp. 37-40, March 2016, Santa Fe, NM.

[23] G. Paul, J. Cardinale, and I. F. Sbalzarini, "Coupling image restoration and segmentation: A generalized linear model/Bregman perspective," International Journal of Computer Vision, vol. 104, no. 1, pp. 69-93, March 2013.

[24] C. Li, C. Kao, J. C. Gore, and Z. Ding, "Minimization of region-scalable fitting energy for image segmentation," IEEE Transactions on Image Processing, vol. 17, no. 10, pp. 19401949, October 2008.

[25] A. du Chene, C. Min, and F. Gibou, "Second-order accurate computation of curvatures in a level set framework using novel high-order reinitialization schemes," Journal of Scientific Computing, vol. 35, no. 2, pp. 114-131, June 2008.

[26] J. Clendenon, C. Phillips, R. Sandoval, S. Fang, and K. Dunn, "Voxx: A PC-based, near real-time volume rendering system for biological microscopy," American Journal of PhysiologyCell Physiology, vol. 282, no. 1, pp. C213-C218, January 2002. 